\title{
A Feasible Molecular Diagnostic Strategy for Rare Genetic Disorders Within Resource- Constrained Environments
}

Maria Mabyalwa Mudau ( $\sim$ mabyalwa.mudau@wits.ac.za)

University of the Witwatersrand

Heather Seymour

University of the Witwatersrand

Patracia Nevondwe

University of the Witwatersrand

Robyn Kerr

University of the Witwatersrand

Careni Spencer

University of the Witwatersrand

Candice Feben

University of the Witwatersrand

Zané Lombard

University of the Witwatersrand

Amanda Krause

University of the Witwatersrand

Nadia Carstens

University of the Witwatersrand

\section{Research Article}

Keywords: Custom-designed NGS targeted panel, genetic testing, rare diseases, resource-constrained environment

Posted Date: September 20th, 2021

DOI: https://doi.org/10.21203/rs.3.rs-857929/v1

License: (c) (1) This work is licensed under a Creative Commons Attribution 4.0 International License.

Read Full License 


\section{Abstract}

\section{Background}

The timeous and accurate diagnosis of rare genetic disorders is critical, as it enables a better understanding of patient management, prognosis and more personalized treatment. A confirmed genetic diagnosis also enables accurate genetic counselling for affected individuals and their relatives by offering options for prenatal and cascade testing. In a resource-constrained environment such as the South African State healthcare system, the challenge is to design appropriate and cost-effective assays that will enable accurate genetic diagnostic services in patients of African ancestry across a broad disease spectrum. Next-generation sequencing (NGS) has transformed testing approaches for many Mendelian disorders, but this technology is still relatively expensive to implement. The paucity of baseline genetic data on African populations adds to the complexity of implementing an appropriate NGS-based service and interpretation of results.

\section{Result}

In the current study, the approach taken aimed at balancing NGS cost, efficiency, data quality as well as diagnostic utility of results. As a proof of concept, we describe a feasible diagnostic strategy for genetic disorders frequently seen in our genetics clinics (RASopathies, Cornelia de Lange syndrome, Treacher Collins syndrome, CHARGE syndrome and other phenotypically overlapping syndromes. The customdesigned targeted NGS gene panel enabled mutation screening for these disorders that range in incidence from 1 in 1000 to 1 in 100000 newborns. Samples were batched during sequencing and analyzed selectively based on the clinical phenotype. This study had an overall diagnostic yield of $54.5 \%$.

\section{Conclusion}

The strategy employed is cost-effective as it allows batching of samples from patients with different diseases in a single run, an approach that can be utilized with rare and less frequently ordered molecular diagnostic tests. The subsequent selective analysis pipeline allowed for timeous reporting back of patients results. This is feasible and can be employed for the molecular diagnosis of a wide range of rare monogenic disorders in resource-constrained environment.

\section{Introduction}

There is limited genomic knowledge for most rare monogenic disorders in African patients as this population is largely understudied $(1,2)$. The paucity of genetic data stems from limited resources with regards to limited clinical genetic services but also limited genomic research and computational expertise, new/relevant technologies and biomedical research infrastructure (3). It is important for African rare disease research and clinical care infrastructure to be developed, as this will aid in the development of appropriate health systems to improve the diagnosis of genetic or rare disorders timeously and accurately on the continent. This benefits patients by enabling a better understanding of their prognosis, 
tailored management and surveillance and more personalized treatment (4). A precise genetic diagnosis also enables accurate genetic counselling for affected individuals and their relatives and offers options for testing at risk family members (5).

For rare monogenic disorders it is challenging to develop diagnostic tests, as each one requires separate research, development and validation which could be very costly (6). In addition, rare disorders have low sample volumes leading to few samples in a batch and/or long times between batches thereby increasing turnaround time. Thus, when developing a diagnostic test for rare disorders, it may be preferable to group them together in one sequencing run to save costs and time (7) especially in resource constrained laboratories.

Next generation sequencing (NGS) technology represents a major breakthrough for molecular diagnostics and is rapidly replacing traditional mutation screening approaches for monogenic disorders (8). It is now possible to screen a large number of genes simultaneously through massively parallel sequencing, thereby significantly reducing both costs and time associated with mutation screening $(9,10)$. However, financial and computational resources, as well as human expertise, are usually extremely limited for NGSbased genetic services in laboratories particularly within low-to-middle-income countries (LMIC) with constrained state healthcare systems $(2,3,11)$.

In South Africa $80 \%$ of the population is served by the State healthcare system, but this system lack sufficient resources to render this very important service, especially for diagnostic of genetic disorders. Limited genetic services are available and clustered mainly in three academic hospital complexes. For patients who do receive a diagnosis, this is mostly a clinical diagnosis by a medical geneticist. There is unfortunately limited or there is no molecular diagnostic testing to confirm their clinical diagnosis, as such testing is frequently not available in the country (12). For most patients in the rural areas, even clinical genetic services are not available.

It is therefore vital to implement a testing strategy that is appropriate and effective to the South African (LMIC) setting. Data analysis for NGS is complex and requires high throughput computational software as well as bioinformatics expertise and despite the decreasing costs of NGS technologies, these technologies remain expensive $(13,14)$. Targeted NGS-based gene panel approaches remained cheaper to implement compared to whole genome or whole exome sequencing (WGS or WES) in diagnostic settings, and have consequently become a standard first tier test for most monogenic disorders (13-16).

For a successful implementation of NGS services for clinical diagnosis, a panel must be optimized for maximum use and a multidisciplinary team of scientists, researchers and clinicians must work together to ensure and promote the success of phenotyping to genotyping to diagnosis of a genetic disorder (Lionel et al., 2018; Liu et al., 2019)

In the current study, a multi-disease NGS targeted gene panel was designed to balance cost, efficiency, turnaround time, data quality as well as clinical utility. As a proof of concept, we describe a feasibility 
evaluation of a diagnostic strategy for a small group of rare disorders, but those considered more frequently seen in genetic clinics.

\section{Methods}

\section{Patient selection and ethics}

Patients with a few clinically distinct groups of disorders; RASopathies, Cornelia de Lange syndrome, Treacher Collins syndrome and CHARGE syndrome were selected to be included in the current study. These disorders represent some of the most common test requests at our facility as assessed by local medical geneticists.

Patients were identified through the genetic clinics of the Division of Human Genetics, National Health Laboratory Service and the University of the Witwatersrand and the Faculty of Health Sciences, University of Pretoria at the hospitals around Johannesburg and Pretoria, Gauteng, South Africa and recruited and consented for the research. This study was approved by the Human Research Ethics Committee (Medical) at the University of the Witwatersrand (M160830) and the University of Pretoria Research Ethics Committee (80/2018). Patient DNA was extracted from whole blood using the salting-out method (17) and DNA concentration and quality was assessed using the NanoDrop ${ }^{\circledR}$ ND-1000 spectrophotometer (NanoDrop Technologies, Wilmington, DE, USA) and Qubit fluorometer (Invitrogen by Thermo-Fisher Scientific, South Africa).

\section{Panel design}

Selection of the genes to be included in the custom-designed targeted panel was informed by an extensive literature review and followed the ClinGen gene-disease clinical validity classification framework (18). Most genes were classified as being definitively associated with the phenotype, but some genes with preliminary evidence indicating possible association with the clinical phenotypes to be studied were also added. particularly. These differential diagnosis genes were particularly added as the molecular epidemiology for the target disorders has not yet been characterised in African patients. The final list of genes to be included in the panel (Table 1) consisted of known genes associated with RASopathies (including Noonan syndrome, Noonan syndrome with multiple lentigines, capillary malformation-arteriovenous malformation syndrome, Costello syndrome, Cardio-facio-cutaneous syndrome, Legius syndrome and Neurofibromatosis), Cohesinopathies (Cornelia de Lange syndrome and related phenotypes), facial dysostoses (Treacher Collins syndrome, Nager syndrome and Miller syndrome) and CHARGE syndrome as well their differential diagnoses. One panel was developed for these disease groups to maximize resources and reduce costs because it allows for batching of different patient groups for testing on one panel. It is cheaper to use one panel rather than three panels for the same number of patients as there are fixed minimum costs on the custom panel depending on the probe region. For example, using Agilent probe design tools, the probe region 1-499kb falls under tier 1 pricing and this was used in the current study. 
Table 1

Genes included in the custom-designed NGS targeted panel, their associated clinical phenotypes and ClinGen association classification

\begin{tabular}{|c|c|c|}
\hline $\begin{array}{l}\text { Clinical } \\
\text { Phenotype }\end{array}$ & Genes & $\begin{array}{l}\text { ClinGen } \\
\text { classification }\end{array}$ \\
\hline RASopathies & $\begin{array}{l}\text { CBL, LZTR1, KRAS, NRAS, PTPN11, RAF1, SOS1, RIT1, SOS2, } \\
\text { RASA1, BRAF, MAP2K1, MAP2K2, SPRED1, NF1, NF2, HRAS, } \\
\text { SHOC2 }\end{array}$ & $\begin{array}{l}\text { Definitive } \\
\text { association }\end{array}$ \\
\hline $\begin{array}{l}\text { Treacher } \\
\text { Collins } \\
\text { Syndrome }\end{array}$ & TCOF1, POLR1C, POLR1D & $\begin{array}{l}\text { Definitive } \\
\text { association }\end{array}$ \\
\hline $\begin{array}{l}\text { CHARGE } \\
\text { Syndrome }\end{array}$ & CHD7 & $\begin{array}{l}\text { Definitive } \\
\text { association }\end{array}$ \\
\hline $\begin{array}{l}\text { Cornelia de } \\
\text { Lange } \\
\text { Syndrome }\end{array}$ & NIPBL, HDAC8, RAD21, SMC1A, SMC3 & $\begin{array}{l}\text { Definitive } \\
\text { association }\end{array}$ \\
\hline $\begin{array}{l}\text { Additional } \\
\text { preliminary and } \\
\text { differential } \\
\text { diagnosis } \\
\text { genes }\end{array}$ & $\begin{array}{l}\text { SF3B4, DHODH, EFTUD2, SMAD4, SMAD5, SMAD6, SMAD7, } \\
\text { EYA1, PDS5A, PDS5B, SCC4, STAG1, ESCO2, AFF4, ANKRD11, } \\
\text { KMT2A, TAF1, TAF6, SETD5, SMARCB1, ARID1B, RRAS, } \\
\text { RASA2, A2ML1 }\end{array}$ & $\begin{array}{l}\text { Limited/Disputed } \\
\text { association with } \\
\text { the clinical } \\
\text { phenotypes. }\end{array}$ \\
\hline
\end{tabular}

The Agilent SureDesign software (version 7.0.2.12) (Agilent technologies, CA, USA) was used to design the targeted panel, with a probe region size of less than $499 \mathrm{kbp}$. Only coding regions of these genes were included, with an additional 10 bases of flanking region added to cover exon-intron boundaries. The probe tiling parameters were edited to ensure full coverage of the genes with low complexity and repetitive regions.

\section{Library preparation and sequencing}

Library preparation was done according to the Agilent SureSelect ${ }^{\mathrm{QXT}}$ workflow, and approximately $25 \mathrm{ng}$ of input DNA is required. DNA is fragmented and the fragments are tagged with adaptors. Tagged fragments were then purified and the desired size was selected using AMPure beads (Beckman Coulter, CA, USA). DNA quality and quantity of the amplicons was assessed using the Qubit fluorometer (Invitrogen by Thermo-Fisher Scientific, South Africa) and the Bioanalyser 2100 (Agilent technologies, CA, USA). The purified amplicons are hybridized to the custom designed capture library. Hybridized amplicons are captured on strepdavidin-coated beads (Thermo Fisher Scientific Inc. MA, USA) then amplified using indexing primers. Indexed libraries are then purified and pooled together in equimolar amounts before sequencing. Pooled libraries (6-10pM) were sequenced in low- (8 samples) and higher throughout (24 samples) batches by utilizing the appropriate MiSeq reagent v2 kits (nano, micro or standard kits) on the Illumina MiSeq Instrument following manufacturer protocols (Illumina, CA, USA).

\section{Variant calling and interpretation}


Read alignment and variant calling was done using the Agilent SureCall data analysis software (version 4.0) using default parameters. The variant call file (VCF) file of each sample was annotated using WANNOVAR (19). Common variants were filtered out using $>1 \%$, minor allele frequency from population databases, clinical significance, in-silico protein effect prediction tools as well as mode of inheritance for each phenotype. Data analysis was targeted, in that only genes pertinent to a specific disorder were evaluated. Clinically significant variants were then classified using the American College of Medical Genetics and Genomics and Association for Molecular Pathology (ACMG-AMP) guidelines (20) to identify putative disease-causing variants. The data analysis pipeline employed in this study is limited to variants smaller than 50bp. Integrative genomic viewer (IGV) was utilized to visualize the coverage depth of the target regions sequenced (21). Sanger sequencing was used to verify the presence of putative diseasecausing variants in patient samples where possible.

\section{Results}

The probe region size of the custom NGS panel was $249.95 \mathrm{~kb}$, containing 3375 probes in total, making the panel appropriately sized to work on smaller benchtop NGS systems. Our custom panel was used successfully to sequence on the Illumina MiSeq system using the nano-, micro- and the standard MiSeq reagent $\mathrm{v} 2$ kits. The coverage obtained per target region ranged from $50 \mathrm{X}-374 \mathrm{X}$.

The panel was used to sequence patients with a clinical diagnosis or suspicion of Cornelia de Lange syndrome $(n=14)$, Treacher Collins syndrome $(n=9)$ CHARGE syndrome $(n=5)$ and suspected RASopathies $(n=60)$. A total of 48 putative disease-causing variants (Class 4 or 5 ) were identified in 88 patients, resulting in an overall diagnostic yield of $54.5 \%$ (Table 2 ).

Table 2

Types of disease-causing variants detected using the NGS targeted panel and the overall detection rate per disorder

\begin{tabular}{|lllll|}
\hline $\begin{array}{l}\text { Disease causing } \\
\text { variant type }\end{array}$ & $\begin{array}{l}\text { RASopathies } \\
(\mathbf{n = 6 0})\end{array}$ & $\begin{array}{l}\text { TCS }(\mathbf{n} \\
\mathbf{9} 9)\end{array}$ & $\begin{array}{l}\text { CHARGE } \\
\text { syndrome }(\mathbf{n}=5)\end{array}$ & $\begin{array}{l}\text { Cornelia de Lange } \\
\text { Syndrome }(\mathbf{n = 1 4})\end{array}$ \\
\hline Missense & 26 & 0 & 0 & 2 \\
\hline Nonsense & 5 & 0 & 3 & 0 \\
\hline Frameshift & 2 & 3 & 1 & 4 \\
\hline Splice site & 0 & 0 & 0 & 1 \\
\hline $\begin{array}{l}\text { Single base } \\
\text { duplication }\end{array}$ & 0 & 0 & 0 & 1 \\
\hline Mutation yield & $55 \%$ & $33 \%$ & $80 \%$ & $57 \%$ \\
\hline
\end{tabular}

Of the disease causing variants identified in this current study, 34 (70.8\%) were previously reported and $14(29.2 \%)$ were novel and not reported in variant interpretation databases such as ClinVar, human genome mutation database $\mathrm{HGMD} \circledast$ and the scientific literature. Novel variants of uncertain significance 
(VUS) were identified in (5/88) $5.7 \%$ of the patients. The putative disease-causing variants identified using the custom panel in our current study were confirmed using Sanger sequencing. The clinically significant variants were also identified in the EFTUD2 and STAG1 genes which were added as part of differential diagnoses, indicating the importance of including the preliminary genes in our panel.

\section{Discussion}

An urgent need exists for African genomic data that could be used to design and implement diagnostic strategies and treatments that are appropriate for patients of African ancestry (11). We present a costeffective strategy for establishing diagnostic testing for monogenic disorders in a resource-constrained environment. Targeted gene panels are still considerably cheaper than other NGS-based methods in LMIC. For this reason, there is an increased interest in using this approach for mutation screening of genetic diseases with relatively limited locus heterogeneity (23). Targeted panels are typically designed for a group of related genes, disorders or phenotypes $(7,24)$. Our approach differs from conventional panel designs as we grouped clinically unrelated disorders in one targeted panel based on the reported need in our genetic clinics to try and achieve batching and costing efficiency. For maximum efficiency, as many genes as possible were included on a panel (based on the sequencing capacity of the lowest panel pricing option) while still allowing for adequate coverage. Selective analysis allowed us to limit analysis and report only to the genes relevant to each patient's clinical phenotype. This helped to streamline the analysis step.

The current study employed a principle of investigating known genes (associated with RASopathies, CHARGE syndrome, Treacher Collins and Cornelia de Lange syndrome) in the custom targeted panel as a point of departure. Selective analysis of the disorders included in the panel had a diagnostic yield of $55 \%$ for RASopathies, which was slightly lower than in a study done by (25) where a RASopathy panel testing identified $68 \%$ of mutations in a central European population. CHARGE syndrome patients had a diagnostic yield of $80 \%$, similar to the $80 \%$ reported by Janssen et al in a Caucasian cohort using whole exome sequencing $(26,27)$. The diagnostic yield of $33 \%$ obtained for Treacher Collins syndrome patients, was lower than the $71.4 \%$ of (28) reported when using WES and Sanger sequencing. In the Cornelia de Lange patients in the current study, mutations were identified in $57 \%$, which is lower than the reported diagnostic yield of $70 \%$ for Cornelia de Lange patients in Germany when NGS gene panel was used (29). The diagnostic yields achieved per disorders studied in the current study are comparable to the literature but are lower in some cases. This likely reflects diversity of mutations in an African cohort. Identification of mutations in known genes could serve as a first line investigation and indicates the great potential this panel has, although all patients may not receive a diagnosis and may ultimately require additional whole exome or genome sequencing. Importantly, some mutations were also identified in the preliminary evidence and differential diagnosis genes, thus justifying their inclusion and providing additional baseline data.

Novel variants of uncertain significance t were obtained in $5.7 \%$ of the patients. This is a relatively low figure, because known genes associated with the disorders tested were analyzed. This still highlights the 
lack of African specific genetic data in public databases which complicate NGS-based testing and data analysis in non-European populations $(30,31)$. This begs the need for researchers focusing on African genomes to collaborate and share their genetic data, so that the uncertainty can be resolved $(32,33)$. Additionally the $29 \%$ of new/novel variants identified in this study provides new insights into African mutation profiles in some more common genetic syndromes that overlap but differ from those in the published literature, and may, in some cases, require local tailored diagnostic approaches.

The putative disease-causing variants identified using the custom panel in our current study were confirmed using Sanger sequencing. This was done in order to validate results before reporting these back to the patients, as this was the first NGS study in our laboratory. The quality of the reads obtained using the targeted panel was good, all had $>$ Q30, and the sequencing coverage depth was above 30x for all the samples, as visualized on the integrative genome viewer (IGV). This validation together with fine tuning of parameters used for NGS data quality control procedure has enabled us to determine when to perform Sanger validation as described in various guidelines for NGS implementation in a diagnostic setting (34-36) and to manage cost further. In our current setting, Sanger sequencing will be used where variant calling is uncertain, such as from homopolymer or poorly covered regions of the genome and for cascade testing in families (37-39).

\section{Conclusion}

The current study demonstrated tthe feasibility and cost-effectiveness of combining clinically distinct diseases on one sequencing panel, followed by selective analysis as a strategic diagnostic tool for these rare heterogeneous genetic diseases in a limited-resourced setting. This strategy has the potential to improve diagnostic service as it allows batching of different tests in one run utilizing the sequencing capacity to minimize costs and reduce turnaround time.

All validated disease-causing variants were reported back to the patients, this gave us the opportunity to improve the decision-making on the management of the patient's disorder. We were also able to provide informed genetic counselling service to the patients. Although the panel was validated on Treacher Collins syndrome, CHARGE syndrome, Cornelia de Lange syndrome as well as RASopathy syndromes, this approach of combining conditions to maximize sequencing capacity and analyzing many rare conditions simultaneously on one test, can be extended to other genetic disorders. This panel was optimized and validated on the Illumina MiSeq platform, but the approach is amenable to smaller benchtop NGS platforms such as the lllumina iSeq, which requires smaller capital investment, as well as other NGS chemistries. As many diagnostic laboratories move towards using NGS in low resource environments, cost-effectiveness should be a high priority due to the number of competing demands in the healthcare sector. There are factors that influence costs and these should be considered when developing NGS based diagnostic tests. Apart from reagents and instrument costs, there are human resource costs, particularly the time of scientists involved in the analysis, data storage costs and logistics, validations of procedures and performing additional confirmatory procedures. The knowledge gained from this study serves as a foundation to develop a more appropriate and cost-effective 
diagnostic testing strategy for monogenic disorders in a laboratory with limited resources thereby providing potential for increasingly effective genetic diagnostic testing to the South African public health care system. Similar value would be expected in other limited resource environments.

\section{Declarations}

Ethics approval and consent to participate: The study was approved by the Human Research Ethics Committee (Medical) of the University of the Witwatersrand (M170761). Informed consent has been obtained from the patients and/or parents, as appropriate. All methods were performed in accordance with the relevant guidelines and regulations

Patients consent for publication: Not applicable

Availability of data and materials: The datasets used or analysed during the current study are available on reasonable request.

Competing interests: The authors declare no competing interests

Funding: This work is based on the research funded in part by the National Research Foundation (NRF) of South Africa (Grant Numbers: 114019 and 106948). The financial assistance of the NRF towards this research is hereby acknowledged. This research was also funded in part by University of the Witwatersrand FRC individual grant (Grant Number: 001.251.8468101.5121105), the National Health Laboratory Service Research Trust (Grant Number. 94656) and South African Medical Research Council (SAMRC) with funds received from the Self-Initiated Research Grant (SIR).

The views and opinions expressed and conclusions arrived at, are those of the authors and are not necessarily to be attributed to the NRF, the SAMRC or the NHLS.

\section{Author contributions statement}

MM prepared the manuscript and designed the next generation sequencing targeted gene panel and utilized it to study genetic aetiology of RASopathies, performed experiments and data analysis. HS contributed to the next generation sequencing targeted gene panel and utilized it to study genetic aetiology of Cornelia de Lange syndrome, performed experiments and data analysis. PN contributed to the next generation sequencing targeted gene panel and utilized it to study genetic aetiology of Treacher Collins and CHARGE syndromes, performed experiments and data analysis. CF, CS and AK was involved in patient recruitment and performed clinical examination/assessments; critically revised the current manuscript and gave important intellectual input. RK and ZL guiding and supervision of the project and also critically revised the current manuscript and gave important intellectual input. NC involved in conceptualizing the entire project, involved with acquisition of data and analysis also critically revised the current manuscript and gave important intellectual input. All authors read and approved the final version of the manuscript 
Acknowledgements: The authors would like to acknowledge and thank the participants and their families as well as the referring doctors.

\section{References}

1. Popejoy AB, Fullerton SM. Genomics is failing on diversity. Nature News. 2016 Oct 13;538(7624):161.

2. Baynam GS, Groft S, van der Westhuizen FH, Gassman SD, du Plessis K, Coles EP, et al. A call for global action for rare diseases in Africa. Nature Genetics. 2020 Jan;52(1):21-6.

3. Musanabaganwa C, Mihigo B, Tumusime R, Uwanyirigira M, da Rocha J, Hayat M, et al. Building Skills and Resources for Genomics, Epigenetics, and Bioinformatics Research for Africa: Report of the Joint 11th Conference of the African Society of Human Genetics and 12th H3Africa Consortium, 2018. Am J Trop Med Hyg. 2020 Jun;102(6):1417-24.

4. Kamp M, Krause A, Ramsay M. Has translational genomics come of age in Africa? Human Molecular Genetics [Internet]. 2021 Jul 8 [cited 2021 Jul 19];(ddab180). Available from: https://doi.org/10.1093/hmg/ddab180

5. Patch C, Middleton A. Genetic counselling in the era of genomic medicine. British Medical Bulletin. 2018 Jun 1;126(1):27-36.

6. Di Resta C, Galbiati S, Carrera P, Ferrari M. Next-generation sequencing approach for the diagnosis of human diseases: open challenges and new opportunities. EJIFCC. 2018 Apr 30;29(1):4-14.

7. Santani A, Murrell J, Funke B, Yu Z, Hegde M, Mao R, et al. Development and Validation of Targeted Next-Generation Sequencing Panels for Detection of Germline Variants in Inherited Diseases. Archives of Pathology \& Laboratory Medicine. 2017 Mar 21;141(6):787-97.

8. Adams DR, Eng CM. Next-Generation Sequencing to Diagnose Suspected Genetic Disorders. New England Journal of Medicine. 2018 Oct 4;379(14):1353-62.

9. Xue Y, Ankala A, Wilcox WR, Hegde MR. Solving the molecular diagnostic testing conundrum for Mendelian disorders in the era of next-generation sequencing: single-gene, gene panel, or exome/genome sequencing. Genetics in Medicine. 2015 Jun;17(6):444-51.

10. Yohe S, Hauge A, Bunjer K, Kemmer T, Bower M, Schomaker M, et al. Clinical Validation of Targeted Next-Generation Sequencing for Inherited Disorders. Archives of Pathology \& Laboratory Medicine. 2015 Jan 22;139(2):204-10.

11. Maxmen A. The next chapter for African genomics. Nature. 2020;578(7795):350-4.

12. Kromberg JGR, Sizer EB, Christianson AL. Genetic services and testing in South Africa. J Community Genet. 2013 Jul;4(3):413-23.

13. Clark MM, Stark Z, Farnaes L, Tan TY, White SM, Dimmock D, et al. Meta-analysis of the diagnostic and clinical utility of genome and exome sequencing and chromosomal microarray in children with suspected genetic diseases. npj Genomic Medicine. 2018 Jul 9;3(1):1-10.

14. Fujiki R, Ikeda M, Yoshida A, Akiko M, Yao Y, Nishimura M, et al. Assessing the Accuracy of Variant Detection in Cost-Effective Gene Panel Testing by Next-Generation Sequencing. The Journal of 
Molecular Diagnostics. 2018 Sep 1;20(5):572-82.

15. Beale S, Sanderson D, Sanniti A, Dundar Y, Boland A. Economics [Internet]. A scoping study to explore the cost-effectiveness of next-generation sequencing compared with traditional genetic testing for the diagnosis of learning disabilities in children. NIHR Journals Library; 2015 [cited 2020 Aug 13]. Available from: https://www.ncbi.nlm.nih.gov/books/NBK373800/

16. Marino P, Touzani R, Perrier L, Rouleau E, Kossi DS, Zhaomin Z, et al. Cost of cancer diagnosis using next-generation sequencing targeted gene panels in routine practice: a nationwide French study. Eur J Hum Genet. 2018 Mar;26(3):314-23.

17. Miller SA, Dykes DD, Polesky HF. A simple salting out procedure for extracting DNA from human nucleated cells. Nucleic Acids Res. 1988 Feb 11;16(3):1215.

18. Strande NT, Riggs ER, Buchanan AH, Ceyhan-Birsoy O, DiStefano M, Dwight SS, et al. Evaluating the Clinical Validity of Gene-Disease Associations: An Evidence-Based Framework Developed by the Clinical Genome Resource. Am J Hum Genet. 2017 Jun 1;100(6):895-906.

19. Chang $X$, Wang K. wANNOVAR: annotating genetic variants for personal genomes via the web. Journal of Medical Genetics. 2012 Jul;49(7):433-6.

20. Richards S, Aziz N, Bale S, Bick D, Das S, Gastier-Foster J, et al. Standards and guidelines for the interpretation of sequence variants: a joint consensus recommendation of the American College of Medical Genetics and Genomics and the Association for Molecular Pathology. Genet Med. 2015 May;17(5):405-24.

21. Robinson JT, Thorvaldsdóttir $\mathrm{H}$, Wenger AM, Zehir A, Mesirov JP. Variant Review with the Integrative Genomics Viewer. Cancer Res. 2017 Nov 1;77(21):e31-4.

22. Watkins NA, Charames GS. Implementing Next-Generation Sequencing in Clinical Practice. The Journal of Applied Laboratory Medicine. 2018 Sep 1;3(2):338-41.

23. Castellanos E, Gel B, Rosas I, Tornero E, Santín S, Pluvinet R, et al. A comprehensive custom panel design for routine hereditary cancer testing: preserving control, improving diagnostics and revealing a complex variation landscape. Scientific Reports. 2017 Jan 4;7(1):39348.

24. Gulilat M, Lamb T, Teft WA, Wang J, Dron JS, Robinson JF, et al. Targeted next generation sequencing as a tool for precision medicine. BMC Medical Genomics. 2019 Jun 3;12(1):81.

25. Čizmárová M, Hlinková K, Bertok S, Kotnik P, Duba HC, Bertalan R, et al. New Mutations Associated with Rasopathies in a Central European Population and Genotype-Phenotype Correlations. Ann Hum Genet. 2016 Jan;80(1):50-62.

26. Janssen N, Bergman JEH, Swertz MA, Tranebjaerg L, Lodahl M, Schoots J, et al. Mutation update on the CHD7 gene involved in CHARGE syndrome. Hum Mutat. 2012 Aug;33(8):1149-60.

27. Ravenswaaij-Arts $C$ van, Martin DM. New insights and advances in CHARGE syndrome: Diagnosis, etiologies, treatments, and research discoveries. American Journal of Medical Genetics Part C: Seminars in Medical Genetics. 2017;175(4):397-406.

28. Fan X, Wang Y, Fan Y, Du H, Luo N, Zhang S, et al. TCOF1 pathogenic variants identified by Wholeexome sequencing in Chinese Treacher Collins syndrome families and hearing rehabilitation effect. 
Orphanet Journal of Rare Diseases. 2019 Jul 15;14(1):178.

29. Braunholz D, Obieglo C, Parenti I, Pozojevic J, Eckhold J, Reiz B, et al. Hidden mutations in Cornelia de Lange syndrome limitations of sanger sequencing in molecular diagnostics. Hum Mutat. 2015 Jan;36(1):26-9.

30. Horak P, Fröhling S, Glimm H. Integrating next-generation sequencing into clinical oncology: strategies, promises and pitfalls. ESMO Open [Internet]. 2016 Nov 18 [cited 2020 Aug 12];1(5). Available from: https://www.ncbi.nlm.nih.gov/pmc/articles/PMC5133384/

31. Pottinger Tess D., Puckelwartz Megan J., Pesce Lorenzo L., Robinson Avery, Kearns Samuel, Pacheco Jennifer A., et al. Pathogenic and Uncertain Genetic Variants Have Clinical Cardiac Correlates in Diverse Biobank Participants. Journal of the American Heart Association. 2020 Feb 4;9(3):e013808.

32. Krause A. New genetic testing technologies: Advantages and limitations. South African Medical Journal. 2019 Mar 29;109(4):207-9.

33. Clift K, Macklin S, Halverson C, McCormick JB, Abu Dabrh AM, Hines S. Patients' views on variants of uncertain significance across indications. J Community Genet. 2020 Apr;11(2):139-45.

34. Aziz N, Zhao Q, Bry L, Driscoll DK, Funke B, Gibson JS, et al. College of American Pathologists' laboratory standards for next-generation sequencing clinical tests. Arch Pathol Lab Med. 2015 Apr;139(4):481-93.

35. Matthijs G, Souche E, Alders M, Corveleyn A, Eck S, Feenstra I, et al. Guidelines for diagnostic nextgeneration sequencing. Eur J Hum Genet. 2016 Jan;24(1):2-5.

36. Hume S, Nelson TN, Speevak M, McCready E, Agatep R, Feilotter H, et al. CCMG practice guideline: laboratory guidelines for next-generation sequencing. Journal of Medical Genetics. 2019 Dec 1;56(12):792-800.

37. Baudhuin LM, Lagerstedt SA, Klee EW, Fadra N, Oglesbee D, Ferber MJ. Confirming Variants in NextGeneration Sequencing Panel Testing by Sanger Sequencing. J Mol Diagn. 2015 Jul;17(4):456-61.

38. Beck TF, Mullikin JC, NISC Comparative Sequencing Program, Biesecker LG. Systematic Evaluation of Sanger Validation of Next-Generation Sequencing Variants. Clin Chem. 2016 Apr;62(4):647-54.

39. Mu W, Lu H-M, Chen J, Li S, Elliott AM. Sanger Confirmation Is Required to Achieve Optimal Sensitivity and Specificity in Next-Generation Sequencing Panel Testing. J Mol Diagn. 2016;18(6):923-32. 